\title{
Enhancing the benefits of group involvement in research
}

\author{
Alice Home ${ }^{1}$
}

\begin{abstract}
Research can facilitate mutual learning, allow participants' voices to be heard, increase practical usefulness of studies and foster empowerment. This paper discusses ways that groups can take part in research, outlines advantages and limits of each and explores strategies for enhancing benefits. This content is illustrated with brief examples from recent research publications and from two longer case studies. Groups and members can be involved as participants or co-producers of research. As participants, they either act as research subjects by contributing data, or as collaborators who are consulted at various times to help keep a study relevant to community issues. Being a subject offers an opportunity to reflect and share views, while collaborators and researchers can learn from working together. Though collaborators can exert influence, they have little control over decisions around focus, design, methods or dissemination of a study. Co-producing knowledge offers community groups more power, learning and empowerment but requires high levels of mutual trust, commitment and persistence. Potential gains and risks increase as involvement intensifies. However, researchers can enhance benefits at any level, by keeping this goal in mind when planning studies.
\end{abstract}

Keywords: groups; community; research; benefits; challenges; groupwork; group work

1. Professor Emeritus of Social Work, University of Ottawa

Address for correspondence: ahome@uottawa.ca

Date of first (online) publication: 3rd December 2021 


\section{Introduction}

Research participation can be beneficial to all involved, when focused on community-relevant concerns and carried out collaboratively. It can allow voices of group members and facilitators to be heard, facilitate mutual learning and enhance the practical impact of researchers' work. Despite this, research publications are limited and do not represent the full diversity of groups, settings, populations and practices in the community. Most of this work has examined certain types of groups, whose development and outcomes are more predictable and easier to measure (Ward 2003). Though some recent studies explore support and empowerment groups, much remains to be done before research publications reflect the full reality on the ground. This paper discusses different ways that groups can be involved in research and explores how benefits can be enhanced for all. We set the stage by reviewing some issues in group work research and by describing some types of groups commonly seen in practice and in the community. We present participant and producer paths to research involvement as well as their advantages and challenges, with examples from recent publications. To explore in greater depth how groups can be co-producers of research, we offer two contrasting case studies from our own work.

\section{Background}

Addressing the well documented gap between practice and research is more challenging in group work, as numerous processes can influence outcome. It is not surprising that most publications describe practice or develop theory. The sparse research literature is dominated by outcomeoriented studies of psychoeducation and certain therapy groups (Macgowan 2014), in which practitioners have considerable control of process and content. This research is useful in many practice settings, where standardized programmes are used in the hope of increasing efficiency or saving time (Preston-Shoot, 2014). It can also provide guidance to increasing numbers of facilitators who, lacking formal group work preparation, gravitate towards simpler, more prescriptive approaches (Sweifach \& Heft-Laporte, 2013). However, this work cannot be applied to the full range of contexts where group work takes place. 
These interventions are not adapted to members' ways of learning in many settings (Preston-Shoot, 2007), nor do they reflect strengthsbased, preventive or social action traditions which have informed practice in marginalized communities (Breton, 1990). With limited research to guide them, practitioners in such settings are left on their own to figure out what works best and why.

To understand this complex issue, it is useful to revisit differences between professionally and peer-led groups. Facilitators of the latter draw their expertise from lived experience with members' shared problem or situation. Ownership and control of these self-help or support groups rests with members (Boyce et al, 2014), though professionals may form the group, act as consultants or participate in meetings. These openended groups usually provide support or information and may engage in advocacy, community education or social action. Facilitators of professionally-led groups draw their expertise from formal education; only rarely have they experienced members' situation. Control remains with practitioners, though the extent varies with different approaches. At one end of the spectrum are psychoeducational groups, which use standardized, time-limited programmes to help members learn skills for managing life problems. An example is a group aimed at teaching atrisk adolescents anger management and social skills, and at improving their school attitude (Dennison et al, 2018). Other interventions mix education and therapy. For example, multiple family groups (MFG) in a low-income community sought to reduce children's behaviour problems and strengthen parenting strategies (Acri et al, 2019). Though most psychoeducational groups make repeated use of the same programme in different settings, some make cultural adaptations. Examples include an MFG group for Chinese families whose children have Attention Deficit Hyperactivity Disorder (Joyce et al 2017), and a group of immigrant Latina survivors of domestic abuse (Marrs Fuchsel 2014).

Adaptation to culture and community is one hallmark of professionallyled groups that focus on mutual aid and/or empowerment. Both these strengths-based approaches are flexible, allowing for adjustment of goals and programme as each unique group develops. While facilitators retain leadership, they focus on attending to and mobilising group processes. Both approaches encourage mutual helping while paying attention to members' environments but differ in some ways. Mutual aid groups typically have a stable membership, focusing on building a safe climate for risk taking, mutual support and demand. These 
features can mitigate worker-member differences, stimulate awareness and foster empowerment, sometimes leading to social action (Shulman, 2006). Empowerment groups can be beneficial for individuals but they emphasise developing leadership, fostering critical awareness and mobilising for collective action. The peripheral facilitator role can be limited to setting up and leading discussion in early meetings, providing resources or information and offering guidance on request (Mullender \& Ward 1991).

\section{Benefits and challenges of research involvement}

In their classic work, Grinnell \& Siegal (1988) encouraged practitioners to become more active in research as participants and/or producers. This section presents ways in which groups and members can be involved in research as participants. In this capacity, they can act either as research subjects who provide data, or as collaborators who contribute expertise at specific times. Advantages and limits of each role are examined and illustrated.

\section{Participating as research subject}

Being subjects in a study allows people to step back from daily lives, reflect on their situation and share their views or experiences. Studies focused solely on outcome offer least benefits, beyond knowing that contributing may help others. Most outcome studies involve therapy or psychoeducational groups and take place in medical or institutional settings. As control and measurement of variables is rigorous, results of such studies are taken seriously in many scholarly circles. However, the findings teach us little about how participants see their experience.

Some researchers have addressed this limit by adding 'open' items to questionnaires. For example, a survey on multiple family groups asked caregivers' what they'd learned, what was most helpful and meaningful. They reported learning behaviour management strategies and identifying parenting areas they could strengthen. Though content on rules, responsibility, relationships and respectful communication was seen as most helpful, the most meaningful aspects were a sense of group cohesion, knowing others understood and feeling they were 
not alone (Acri et al 2019). These findings suggest that some structured interventions can bring benefits like those observed in mutual aid and self-help groups. Simply asking 'Anything else to add?' opens a space for self-expression. Participants in a survey on special needs adoptive parenting replied by sharing personal experiences which could be used in advocacy and media work. One stated 'We are the proudest parents in the world. We all stick together and advocate for our kids continuously. I would choose adoption over and over' (R13). Another warned: 'We have no idea what parenting these kids is like ... and when we look for help, we're told we're on our own' (P9).

Qualitative methods involving interviews can elicit data that might not emerge otherwise. One study sought to learn how group process facilitates change and what motivates abusive men to keep attending (Chovanec 2014). Interviews with members and facilitators revealed that learning new things helped keep men engaged, while hearing others' stories motivated change. These findings confirmed that open educational groups are a good fit for this population, and presence of men further along in treatment can help those just beginning. This insider information could lead to adjustments that would improve outcomes for future members. Interviews offer research subjects a chance to reflect on experience, become aware of issues and explore new ideas. For example, a peer facilitator described challenges encountered in groups of parents whose children had Autism Spectrum Disorder (ASD). The researcher later mentioned that some of these problems occur commonly, as they reflect the complexities of group leadership. The facilitator's response was that peer leaders need access to training. The researcher shared this with group work students, suggesting it would be a practical way to lend support. Listening to respondents can stimulate researcher learning. In this study, hearing about difficult situations and innovative strategies gave the researcher new respect for the courage, resilience, skills and creativity of peer group leaders. Similarly, a graduate assistant came to appreciate the resilience of mothers whose children have ADHD. This prepared her for working in the school system, which often blames mothers for their children's behavior (Home 2014a).

Research interviews conducted in a group context can elicit rich data while increasing participants' learning. Focus groups bring together people in similar situations to share views on an issue with which 
they have experience or expertise. To encourage equal participation while reducing 'groupthink', participants reply in turn to a limited number of questions. Others may respond by agreeing with, adding to or challenging viewpoints expressed (Patton, 2010). For example, adolescent Latino/a survivors of intimate partner violence (IPV) were asked to share what they'd gained from a mutual aid group. They reported increased empowerment and self-esteem, along with lower depression and isolation. They highlighted the group's role in enabling a safe home away from home and shared insights on the impact of Latino culture impact on their experience (Molina \& Chapple, 2017). Another benefit of group interviews is that participants with low power or status may feel more comfortable expressing themselves when surrounded by peers. One study used focus groups to learn about of girl fighting and dating violence while raising awareness of the issue. Researchers created a safe environment for expressing diverse views, then shared findings promptly with school personnel, thereby ensuring that those who could make changes heard participants' voices (Letendre \& Rankin Williams, 2014)

Group interviews can take different forms. In 'brainstorming sessions', recent attenders of open adoptive parent support groups were asked to generate statements describing an effective group. Findings included practical suggestions. Participants stressed that the facilitator should be an adoptive or foster parent who has received facilitation training. They added that participation training could be provided to new attendees, a strategy which might attract and retain members (Miller et al, 2018). They also pointed out that many parents cannot attend without their children, suggesting allowing space for young adoptees, offering them concurrent groups or holding on-line groups for parents (Ibid). Group interviews can be used alone or in conjunction with other data collection methods. A United Kingdom programme for prospective adoptive parents had two unique features: it took place several months after a child was placed, and 'trainers' were themselves adopters (Selwyn, del Tufo \& Frazer, 2009). Facilitators met in focus groups, and parents and agency staff were interviewed. Combining different types and sources of data produced information that had not emerged from studies. For example, parents described an interplay of personal learning, family change and the group experience. Like participants in the previous study, they stressed the importance of facilitators' being insiders: 'I was 
exhausted with explaining to people who haven't experienced it. .... It was such a relief to find that these people knew'. (Ibid, p. 38).

Though research subjects can benefit from taking part in studies, marginalized populations can be inadvertently excluded. For example, an analysis of six focus group studies with people who had intellectual disabilities (ID) found most participants had mild to moderate ID (Wright, 2017). The voices of those with complex needs were not heard. Another problem is that people who gained little from being research subjects in the past may avoid future participation. Their concerns are best expressed in an altered French verb declension: 'I participate, you participate, s/he participates, we participate, you participate, they profit'. These issues have led researchers to develop other paths to involvement which could spread the benefits more evenly.

\section{Participating as research collaborator}

Collaborative approaches seek the input of community groups, to ensure studies are relevant to community concerns and stay on course. Instead of seeking community involvement only to recruit subjects or provide publicity, researches consult community groups at various times in a study. This can allow the latter some influence on focus, data collection and dissemination, though the researcher retains final decision-making power. Roles and consultation arrangements are negotiated at the outset, which lets collaborators assess if this is a realistic commitment for them (Home 2014). Collaboration is usually carried out by an advisory committee, which represents a local community or group impacted by the research problem. These committees offer input and feedback based on experience with or knowledge of the issues or context.

An example is a study which sought to learn how employed mothers of children with ADHD saw their multiple roles, and to identify factors that influence this. The researcher, who had experienced this life situation, approached a national parent organization to explore collaboration. Given the study's relevance to its mission and to member needs, the organization asked a parent and a professional board member to act as advisory committee. The members provided input on all important decisions, helped develop a survey questionnaire from interview data and co-presented a research poster at the organisation's international conference (Home 2014). This collaboration proved beneficial to all 
involved. The committee helped the researcher avoid mistakes, tailor the study more closely to community needs and extend practical usefulness of the work. This experience also helped the researcher. She felt less alone and more solid making decisions, knowing they were grounded in community experience. Collaborating also helped strengthened links between her teaching, research and practice. She used the poster in the classroom to illustrate practice-research, and in a mutual aid/ empowerment group for mothers of children with ADHD co-facilitated with a practitioner. At the group's request, the professional attended one meeting, where he fielded questions and connected members with his organization's local chapter.

Committee members were asked what they had gained and what they found challenging. The professional, a psychologist specialized in ADHD, found this a refreshing change from clinical work. He saw the unique value of qualitative methods while discovering their complexity. When learning new skills, both committee members worried 'Do I know my stuff?' before seeing how invaluable their contributions had been. Both sometimes had difficulty finding little bursts of time when they were needed, given other life and work commitments. The parent, a consultant in the school system, appreciated working across the boundaries separating families, scholars and practitioners. Study results validated his observations, deepened his understanding of challenges facing user groups like his but left him disappointed to find no quick solutions. He noted the recognition that came from co-presenting the poster, as the hard work of peer leadership is so rarely noticed. He added that this collaboration increased his community group's credibility in scholarly and professional circles (Ibid).

This project included feedback sessions in four regions of Canada, to be hosted by local chapters. Goals were to offer parents, professionals and community groups the opportunity to hear and give input on findings. As some hosts living with ADHD might face organisational challenges, the researcher prepared a clear plan which could be adapted to local needs. Summary of findings was distributed to all 100 attendees, posted on the website and published in newsletters of other parent-led groups. One feedback session stimulated innovative new research. Peer leaders of two groups, serving parents of children with ASD and Foetal Alcohol Spectrum Disorder (FASD), noted similarities of family experiences. They suggested that parents, professionals and researchers 
concerned with invisible disabilities should work together rather than compete for limited resources. This led to a study of facilitator views on groups for parents of children with ADHD, ASD or FASD. Findings of that study raised important issues, such as gaps between group format and the needs of families living with lifelong disabilities. Participants saw long-term, open groups as critical 'to stay[ing] connected when things go wrong', as parents are welcomed whenever they need 'an anchor in a stormy family life' (Home 2015, p. 191). They also underlined the camaraderie and empowerment that these peer-led groups bring, while worrying about their precariousness from insecure funding and leadership renewal issues (Ibid).

\section{Producing research knowledge}

Though collaborating can allow community groups some influence, much depends on the researcher, who controls how input will be used, as well as the extent, purposes, methods and timing of consultation. Producing knowledge can bring more benefits but groups and practitioners often lack the time, expertise and access to resources needed to go it alone. Co-producing with university faculty is a more realistic option (Macgowan, 2014). Community-based (CBR) and action-research (AR) approaches can facilitate this. The former seeks to engage community groups in co-creating context-relevant knowledge (Tremblay \& Oliveira Jayme, 2015), while AR aims at bridging the gap between knowing and acting (Morton \& Hohman, 2016). Both strive to foster sustainability of change by building capacity and promoting community ownership (Wood \& McAteer, 2017). These laudable goals are difficult to apply. Bidirectional knowledge sharing requires a climate of mutual trust, respect for diverse knowledges, openness of all parties to learning and their participation in decisions throughout the research (Ibid). To be mutually beneficial, studies must focus on problems relevant to the community, seek knowledge that can be used for social action, employ varied data collection methods and disseminate findings where and when they will make a difference (Wood et al 2015). Groups can play a central role in co-producing research, via participatory data collection and/or as part of university-community teams. However, only a few publications examine the processes involved or the ways in which such teams work.

One study explored how six practitioners experienced group 
practice with survivors of intimate partner violence by asking them to reflect and share knowledge in inquiry groups (Morton \& Hohman, 2016). Researchers opened each session but members helped frame questions, manage discussion and foster equal participation. Another study examined homeless youths' experiences co-producing an audio documentary. Goals were to engage them in the research process, while they collaboratively told their stories of strength and resilience (Kelly 2015). The youth group analysed recordings and productions, selecting those which best embodied their experience. The facilitator empowered the group to make its own decisions, by providing support, resources and information. Findings showed members contributed their talents, acquiring technical, project management and group skills. They participated actively in data collection, analysis and dissemination (Ibid). These positive outcomes were facilitated by group cohesion and a strong culture, as demonstrated in members' accountability to the project and each other (Kelly \& Hunter 2016).

Some studies mix participatory with traditional methods. One explored participants' experiences in a project which brought together youth of varied minority descent for intergroup dialogue (RichardsShuster \& Aldana, 2013). Intervention goals were to stimulate discussion of race and racism while supporting knowledge and skill development. The research aimed to showcase youth's voices and identify programme moments attached to their learning. Data were collected by a research team of youth 'alumni' and university members, as well as by traditional means (survey, focus groups, interviews). Youth reported gaining skills in leadership and communicating across difference. They felt more adept at creating change, and increased their understanding of privilege and oppression. Having learning from group discussions how racism affects peers, some spoke out against it in their daily lives (Ibid).

\section{Co-producing research: Two case studies}

There is ample literature describing conditions for co-producing knowledge and obstacles to success, but few publications discuss details. Two case studies led by the author in different contexts and eras provide specific examples of challenges and strategies. The first was a local community-led project while the second was a national 
community-university partnership.

\section{The respite care project}

This action-research was initiated in the 1980s by community workers in an inner-city Quebec neighbourhood. They noted that many foster placement requests came from one-parent families, exhausted from caring for young children in a context of poverty. Unable to afford scarce day care yet fearful of approaching social services, many delayed help seeking until their situations were untenable. The workers formed a collective with local women and started a community respite care service for needy parents. Care-giving families met monthly to support and learn from each other, infrequent open meetings were held for carereceivers and occasional social gatherings were open to everyone (Home $\&$ Darveau-Fournier 1995). After a year, the Collective approached the researchers about working together to systematise past work, add evaluation and prepare a grant application. This was an opportunity for the researchers to get involved in preventive, empowerment-oriented work compatible with their values.

A slow process of partnership development began, based on an unspoken understanding that mutual trust and respect were paramount. A research team member attended Collective meetings and vice versa, allowing sub-teams to know each other and identify respective knowledges. When preparing the grant application, researchers built on the Collective's work and consulted them frequently. Initial goals were reformulated in specific terms and a theoretical framework was written around practice observations. The evaluation plan addressed both process and outcome, used diverse data collection methods and included a formative component to allow ongoing adjustments. Some administrative tools were adapted for research use, and the Collective reviewed all new instruments to improve fit with local context. For example, an added networking item asked care receivers: 'Who could help you with big cleaning jobs?'. These strategies demystified the research process, strengthened Collective members' confidence in their expertise, while securing their ongoing engagement in the research component (Ibid). They were awarded a three year demonstration project grant, on condition that the researchers be responsible for evaluation. 
Findings confirmed that respite care was relevant to community needs, with low-income, single parents making up 75\% of users. The latter valued this informal, accessible service, some saying they had never felt comfortable asking for help previously. Though that service may have prevented some foster placements, the project had a modest impact on care-receiving families. They felt more rested and some saw short-term improvements in child behaviour or relationships. Few parents used the service more than twice, group meetings were sparsely attended and there was scant evidence of networking change. However, empowerment outcomes of the project were more pronounced. Some service users became care-givers, a few joining the Collective. Workers noticed improved peer-helping skills among care-givers, and observed more critical reflection and attention to group process in meetings. Empowerment and learning benefits were most prominent among Collective members. Their community commitment grew, as did their organizational and research competencies. New leadership and negotiating skills were demonstrated when they obtained funding to continue the respite care service after the grant ended, a rare event in demonstration projects. Finally, theirs was one of five Quebec projects selected for a video on innovative, community responses to family needs. This video, which featured interviews with the Collective and practitioners, was used for many years to teach preventive and strengthsbased practice.

Researchers benefitted from this project in many ways. Hands-on involvement made them better known and increased their credibility outside the university. Their teaching was strengthened by project examples and classroom presentations of Collective members. Graduate assistants in the project found creative ways to integrate practice and research. For example, one acted as Santa's elf-clad helper at an informal gathering, making her more approachable when she later did research interviews. However, these benefits did not come without costs. Though some challenges of non-traditional research have been known for decades, two have been articulated in recent literature: wide power inequities between universities and communities and vast differences in their respective knowledge cultures (Tremblay \& Hall, 2014).

Academics' power lies in their status, formally recognized expertise and access to grants. Though these resources can be useful to communities, universities and funders can withhold support or impose 
conditions that impede meaningful participation. Communities' power lies in their invaluable knowledge of local needs, culture, and networks. However, not all communities recognize this expertise and many lack the confidence or skills to share it (Wood \& McAteer, 2017). As the community controlled this project from the outset, researchers focused on mobilising the Collective's knowledge and ensuring their ongoing participation in research decisions. Strategies included providing point-form summaries prior to meetings and using clear language to explain technical terms. Aware of the potential impact of role and status differences, they adjusted their work schedules to fit the Collective's availability and attended informal project gatherings.

Knowledge culture differences can be subtle yet create powerful obstacles. Universities have long valued traditional research which leads rapidly to publishable results, so their norms, tangible supports and reward systems are not aligned with participatory approaches (Kasi \& Yorks, 2010). Community knowledge cultures reflect a history of oppression and disadvantage which can translate into mistrust. If their capacity has been co-opted in the past, community groups may be wary of joint projects (Kearney, 2015). Differences in priorities can arise as well, reflecting divergent organizational mandates. In this project, researchers understood the Collective's focus on respite care, though they would have put more emphasis on group services. Having to prioritise evaluation while promoting empowerment meant the design had to be systematic yet context-specific and unobtrusive. Neither a control nor comparison group design was acceptable, given the Collective's priority on providing respite rapidly to all needy families. As this service had been operating for a year, pre-and-posttest designs were precluded, as was tracking foster placement rate change (Home $\&$ Darveau-Fournier 1995). The researchers' methodological compromise featured several methods of data collection and analysis, triangulating information from multiple sources.

The community impact of knowledge culture differences became clear when post-grant funding was made available only for the respite care service. This decision, which likely reflects lower societal value attached to community learning and capacity-building, left the Collective on its own to monitor and develop its work. A second issue appeared when the demonstration grant programme was terminated, blocking a simple funding route for modest, community-led initiatives. 
Though multi-year federal support became available, gaining access increasingly required joining large, university-led research projects. Academic knowledge culture later had an impact on the researchers. A peer-reviewed journal quickly rejected an article citing the lack of a control group. The resulting delay in scholarly publication had repercussions a few years later. Another university denied the author's promotion, as her grant amounts and number of peer-reviewed publications were deemed insufficient. On appeal, the School's director explained that this large grant would not have been awarded without the researchers' involvement, and that taking a sub-grant reflected commitment to keeping the project community-led. He added that developing trust, consistently involving partners and following their pace slows community research. This quality versus quantity argument was successful.

\section{Working together for success: A knowledge sharing project}

The second project was more complex, differing from the first in many ways. Though many ideas came from the community, it was the researcher who developed the partnership, obtained a grant and led the work. This project originated with the author's small study of different stakeholders' views on parenting adopted children with disabilities. During interviews and presentations, she was urged to share findings widely. A public outreach funding programme sought to increase access to research, foster multidirectional knowledge sharing and build connections with users. To explore relevance and feasibility, the author consulted five key informants: an agency director, a social worker, a parent association coordinator, a policymaker and an adoption council board member. Noting that disability and child welfare communities rarely collaborate, they suggested bringing together parents, professionals and community groups to learn from researchers and each other. The author formed a community-university partnership with a disability scholar, a national and provincial adoption council, an adoption agency and a parent-led support group. Three team members had adopted children with special needs, and a fourth was grandparent of a child with a disability. Their project sought to share research knowledge with diverse users, seek their input, foster cross-role collaboration and make the work widely available in the community 
(Home et al 2015). Two main activities were proposed: invitational workshops on parenting children with special needs, and creating varied, accessible documents for community use. The two workshops would take place in the autumn of 2012, in the same regions where the original study had taken place.

Upon receiving a dissemination grant, team members met to plan the workshops and fine-tune role distribution. The workshop plan featured two research presentations (on study findings and advocacy), a networking lunch, mixed-role discussion groups and a plenary. To foster engagement and recognize partner expertise, regional committees adapted the core format to local context, generated an invitation list and led one workshop. Several measures were designed to reduce external and internal obstacles to participation. These free events were scheduled for Saturdays, with all costs covered for parent study participants. Invitations emphasised the importance of lived experience to allay concerns around valued types of knowledge. To reduce the impact of status and ensure participants felt safe, name tags indicated first names only and confidentiality was assured. Regional teams recognised different types of expertise by asking local organizations to bring display material and by inviting group coordinators to host lunch networking tables. Context-specific measures were added. Translated invitations and handouts welcomed the Franco-Ontarian minority in Ottawa, where the plenary and one discussion group were conducted bilingually. As Vancouver Island is home to many First Nations, the Victoria team reached out to indigenous stakeholders and offered a group on culture and disability. Sixty parents, professionals and representatives of diverse groups and organizations attended.

Three months later, an independent evaluator interviewed a contrast sample of 15 attendees from both workshops. They were asked about their learning, any changes made and most helpful activities. Findings showed that workshop goals were largely attained. Discovering common concerns, and learning/sharing across boundaries were the most important gains. Parent reported reduced isolation, normalisation of their experience and realisation that professionals' options were limited by policy and budget issues (Ibid). As summed up by the national council board member: 'social workers feel frustrated because they cannot provide the support that they know these families need. I've been there. I've been in a policy position' (Home 2013b. Professionals 
gained respect for families' strength and resiliency, who must fight for every crumb of help. Workshop participants became aware of the crucial role of peer-led support groups, whose very survival was imperilled by inconsistent support. Interviewees agreed that the most helpful activities were mixed role discussion groups and an open, respectful climate which 'recognised all stakeholders equally as experts in their own right' (Home et al 2015, p. 31).

The final project goal was to disseminate this knowledge to a wide range of community stakeholders. Victoria presentations and the plenary were filmed, as were interviews with a parent, a policy maker, a practitioner, and coordinators of two parent associations. From this material and group-verified discussion summaries, the team created a video series designed to help reduce parent isolation and raise public awareness. As user groups had requested practical tools on parenting challenges and strategies to manage them, the team developed short monographs on three workshop themes. One monograph was penned by the $\mathrm{BC}$ graduate assistant who led a discussion group on culture and disability. Researchers prepared drafts and partners provided feedback, imagining they were exhausted parents or overworked professionals. Writers balanced information with accessibility, using a common outline. A graphic designer prepared Web and print versions, to ensure that the monographs would be accessible and engaging for all readers. One video and monograph were translated for French-speaking users. The national adoption council made them available at no cost on their website, while other partners promoted them via their networks and identified strategic organizations for hard copies. Informal feedback suggested that these documents were useful for varied purposes, from parent learning to professional development. The author, national adoption council director and two parents participated in national media interviews. Academic dissemination involved researchers and two community partners presenting at anational conference and coauthoring a peer-reviewed article (Home et al 2015).

All those involved benefitted from this project. Study participants received the videos and monographs, allowing them to see the value of their knowledge and know that it was being shared nation-wide and beyond. Study parents who attended workshops gained more. One revealed 'I had this overwhelming, heartfelt feeling of just being heard' (Home 2013b) and a couple got away together for the first time in the 
5 years since their complex child arrived. Professionals and providers discovered how inadequate respite support was, on learning 'it's up to us to find the respite worker...There's no list. You can't have a teenager, they'll eat them up for supper'. (Home 2013a, p. 6). A parent study participant added 'If we had the right supports in place, I think families and kids would thrive. That's what we all want, right?' (Home, 2013b). During the closing plenary, the adoption council board member pleaded with professionals to "listen to the parents. They are the people who know what their children need, and they are the best advocates. But they need help advocating' (Ibid).

Research team members reflected on what they had gained from their experience. This was the first time that the author's work was made available to those who needed it. When developing the videos, she learned techniques for sharing her research in new ways. She also learned how to manage a complex project involving multiple institutional and community partners in different locations. The disability scholar deepened her understanding of parents' challenges, admired their eagerness to learn and appreciated sharing practical advocacy knowledge. The agency partner, who was also a graduate assistant, saw how her work attracting diverse service providers and organisations to the $\mathrm{BC}$ workshop helped break down silos. She learned qualitative and community research skills and rejoiced that her student research was of practical use. The adoption council board member was disappointed to see so little change in the years since her organisation's surveys, yet she was encouraged to see stakeholders working together. Learning that it was crucial to invest in peer to peer support was reassuring for the parent group leader, who found herself responding to entire families falling into extreme financial and emotional distress. Lastly, the national adoption council benefitted in an unexpected way. With support from the author, this partner carried out a survey to verify and deepen understanding of the qualitative study findings. This strengthened the executive director's research skills. She later presented the results at national professional conference, which increased her organisation's credibility.

These benefits did not come easily. Full team meetings were impeded by vast distances, budget limits, members' organizational priorities and commitments. The parent group coordinator participated in planning and holding one workshop, but later involvement was limited by family 
and support group crises. The author's professor emeritus status allowed her to commit full time to the project, unlike regular faculty who must squeeze research into their heavy workload. However, her relocation in another province and reorganisation of the university research service complicated communication, coordination and management. For example, the first workshop was organised by a graduate assistant, as grant guidelines and budget precluded hiring an event manager. Despite getting regular supervision at a distance, this inexperienced student became overwhelmed and quit two days prior to the workshop. It went ahead only because the national partner mobilised volunteers rapidly and the School's director provided extra support. Management challenges were exacerbated by bureaucratic university policies, which contrasted starkly with community partners' straightforward ways of working. Team tensions occurred when members had to provide detailed receipts for minor expenses. Rigid procedures interfered with commitments to workshop participants. Lacking a letter specifying her role and itemising costs, a peer group leader was denied reimbursement of parking expenses. For one couple, even this letter was insufficient. The author had to explain repeatedly why these study participants could attend only if a trusted, trained family member provided care for their child.

\section{Concluding thoughts: How can research involvement become more beneficial?}

These examples and case studies show that gains increase as research involvement intensifies. However, it is possible to enhance benefits at any level by planning studies with this goal in mind. Research subjects will gain more from studies that explore process as well as outcome, include open questions and employ mixed data collection methods. If the latter include interviews, participants have more space for selfexpression, and those done in a group context add the opportunity to learn from others. Regardless of type of study, having prompt access to findings in an accessible format can bring immediate, practical benefits. When results are shared with the community, these gains are extended to others in similar situations and to professionals who can advocate for change.

Collaboration offers an opportunity for community groups to provide 
input without a heavy time commitment. As this role is advisory, however, they may want to assess the extent to which researchers will seek and use their input when making important decisions. Positive prior connections can help. For example, the author's credibility as a parent 'insider' and member of an ADHD community group helped the latter feel more secure appointing an advisory committee. It is important to take time near the beginning of any collaborative project to discuss any concerns or questions and to agree on consultation arrangements. Throughout the study, researchers need to be open to learning from the advisory committee, even if not all suggestions are acted on.

Many of these same principles apply when groups co-produce knowledge with researchers, except that the stakes are higher for both. A Canadian study of 20 diverse community-university partnerships concluded that early investment in relationship building is critical, as 'benefits to the community accrue in direct proportion to the quality, longevity and trust developed' (Tremblay \& Hall, 2014, p 402). Cutting corners at the beginning may be tempting but can undermine community partners' engagement, deprive others of their expertise and reduce what they gain from involvement. Funding timetables can be an obstacle unless potential partners know each other. As the researcher had interviewed most key informants for the adoptive parenting study, she was able to consult them, negotiate roles and establish a partnership in the month available before the grant application deadline. However, a community group that helped shape the project could not get partnership approval in time. Instead, their director attended the workshop and was interviewed for the video series.

An adequate development phase allows potential partners to clarify what they will be expected to do so they can weigh risks and benefits. Community research can be a good fit for faculty committed to social justice provided they are aware of possible career costs. Though universities profess openness, advancement comes to those subscribing to a culture of productivity, measured in numbers of citations, peerreviewed publications and graduate students, as well as in amounts, quantity and prestige of grants obtained (Larochelle et al, 2020). Community groups considering partnership need to remember that they may be pressured to redirect time to issues considered more urgent or central to organizational priorities. Another use of this development phase is to give partners a chance to identify each other's strengths and 
anticipate any challenges that may arise. This work will allow them to devise strategies to circumvent likely obstacles, and quickly address unexpected snags that crop up later.

Applying a few basic principles consistently will help distribute benefits of research more evenly. Researchers who are transparent, demonstrate respect and a desire to learn will be more likely to earn the trust of others involved. A climate of safety is essential if partners and participants are to engage fully, especially if they will be taking a collaborative or co-producer role. Researchers need to keep listening and using others' input throughout the study or project. Finally, a strong commitment to social justice will help in translating these principles into practice but it is not enough. Flexibility and creativity are also required if research is to be more beneficial to all.

\section{References}

Acri A., Hamovitch, E., Lambert, L., Galler, M., Parchment, T. and Bornheimer, L. (2019) Perceived benefits of a multiple family group for children with behavior problems and their families. Social Work with Groups, 42, 3, 197212, DOI: 10.1080/01609513.2019.1567437

Boyce M., Seebohm, P., Chaudhary, S., Munn-Giddings, C., and Avis, M. (2014). Use of social media by self-help and mutual aid groups. Groupwork, 24, 2, 26-44. https://doi.org/10.1921/gpwk.v24i2.784

Breton, M. (1990) Learning from social group work traditions. Social Work with Groups. 13, 3, 21-34.

Chovanec, M. (2014) The power of learning and men's stories in engaging abusive men in the change process: Qualitative study across programs. Social Work with Groups, 37,4, 331-347. DOI: 10.1080/01609513.2014.901207

Dennison S., Daniel, S., Gruber, K., Cavanaugh, A. and Mayfield, A. (2018) A leadership training group for at risk fifth $\&$ seventh graders: Results from a brief strength-based group program. Social Work with Groups, 41, 3, 181-197. DOI: 10.1080/01609513.2017.1330170

Grinnell, R. and Siegal, D. (1988) Social Work Research and Evaluation. (2nd ed.). Itasca: Peacock

Home A. (2013a) Ways forward. Chapter 4 in the video series Special Needs Parenting: Working Together for Success. Retrieved from http://www. adoption.ca/special-needs-parenting. 
Home, A. (2013b) What's going on? Disentangling children's disabilities and getting the right support. Monograph 1 in the series Special Needs Parenting: Working Together for success. Retrieved from http://www.adoption.ca/uploads/ Image/Monographl-Alice-FINAL.pdf

Home, A. (2014) Research with and about groups: Overcoming obstacles to creativity and solidarity. in V. Roy, V., G. Berteau and \& S. Genest Dufault (Eds.). Strengthening Social Solidarity Through Group Work: Research and creative practice. London: Whiting \& Birch (pp 195-210)

Home, A. (2015) 'Without them, I probably wouldn't be on this planet': Benefits and challenges of groups for parents of children with disabilities. in W. Pelech, K. Ring and S. Larocque (Eds.). Unity in Diversity: Embracing the spirit of group work. London: Whiting \& Birch (pp 185-201)

Home, A., Carter, I., Scarth, S. and Warren, R. (2015) Working together in special needs parenting: An innovative research dissemination project. Social Work and Social Sciences Review, 17, 3, 6-19. https://doi.org/10.1921/swssr.v17i3.794

Home, A. and L. Darveau-Fournier (1995). Respite child Care: A support and empowerment strategy for families in a high-risk community. Prevention in Human Services. 12, 1, 69-88

Joyce L., Ma, C, Kelly Y., Lai E. and Wan S. (2017) Multifamily group Intervention for Chinese parents and their children with Attention Deficit Hyperactivity Disorder in a Chinese context. Social Work with Groups. 40, 3, 244-260. DOI: 10.1080/01609513.2015.1132028

Kasi, E. and Yorks, L. (2010). 'Whose inquiry is this anyway?' Adult Education Quarterly, 60 ,4, 315-336. https://doi.org/ 10.1177/0741713609347367

Kearney, J. (2015). Challenges for community engagement: An Australian perspective. Educational Research for Social Change (ERSC), 4 , 1, 26-39. https:// www.researchgate.net/publication/280009803

Kelly, B. and Hunter, M. (2016). Exploring group dynamics in activity-based group work with young people experiencing homelessness. Social Work with Groups. 39, 4, 307-325. DOI: 10.1080/01609513.2015.1061962

Kelly, B. (2015) Using audio documentary to engage young people experiencing homelessness in strengths-based group work. Social Work with Groups. 38,1, 68-86. DOI: 10.1080/01609513.2014.931665.

Larochelle, C et al (2020). Publish or perish: Women in research call for an end to systemic discrimination. University Affairs, Oct-Nov 2020.

Letendre, J. and Rankin Williams, L. (2014) 'I hear you': Using focus groups to give voice to adolescent girls' experiences with violence. Social Work with Groups. 37:2, 114-128. DOI: 10.1080/01609513.2013.823832.

Macgowan, M. (2014) Research! What we can do to advance it in social work 
with groups. in V. Roy, G. Berteau et S. Genest Dufault (Eds). Strengthening Social Solidarity through Group Work: Research and creative practice. London: Whiting \& Birch (pp. 211-225).

Marrs Fuchsel, C. (2014) Exploratory evaluation of Sí, Yo Puedo: A culturally competent empowerment program for immigrant Latina women in group settings. Social Work with Groups. 37, 4, 279-296. DOI: 10.1080/01609513.2014.895921

Miller, J., Sauer, C., Bowman, S., Thrasher, K., Segress, M. (2017). Conceptualizing adoptive parent support groups: A mixed-method process. Adoption Quarterly 26,1, 41-57.

Molina, O., \& Chapple, R. (2017). A mutual-aid group for Latino and Latina adolescent survivors of parental intimate partner violence. Groupwork. 27, 1, 66-86. https://doi.org/10.1921/gpwk.v27i1.1097

Morton, S \& Hohman, M. (2016). 'That's the weight of knowing': Practitioner skills and impact when delivering psychoeducational group work for women who have experienced IPV. Social Work with Groups. 39, 4, 277-291. DOI: 10.1080/01609513.2015.1052915

Mullender, A \& D. Ward (1991). Self-directed Groupwork: Users Take Action for Empowerment. London: Whiting \& Birch.

Patton, M. (2014). Qualitative evaluation and research methods. (4th ed.). Newbury Park, CA: Sage.

Preston-Shoot, M. (2007). Effective groupwork (2nd ed.). Basingstoke: Palgrave. Preston-Shoot, M. (2014). Some ethical and legal challenges in researching groupwork practice. in V. Roy, G. Berteau, and S. Genest Dufault (Eds.) Strengthening social solidarity through group work: Research and creative practice. London: Whiting \& Birch. (pp 226-244)

Richards-Schuster, K \& Aldana, A. (2013) Learning to speak out about racism: Youths' insights on participation in an intergroup dialogues program. Social Work with Groups. 36, 4, 332-348. DOI: 10.1080/01609513.2013.763327

Shulman, L. (2005). The skills of helping individuals, families and groups. (5th ed). Itasca: Ill. Peacock.

Selwyn, J., del Tufo, S. \& L. Frazer (2009). It's a piece of cake? An evaluation of an adopter training programme. Adoption and Fostering 33, 1, 30-41.

Sweifach J. \& Heft LaPorte, H. (2013) Assessing use of the standards for social work practice with groups by school social workers: A national study. Social Work with Groups. 36, 2-3, 130-144. DOI: 10.1080/01609513.2012.745109

Tremblay, C., \& Hall, B. (2014). Learning from community-university research partnerships: A Canadian study on community impact and conditions for success. International Journal of Action Research. 10 ,3, 376-404. https://doi. 
org/10.1688/IJAR-2014-03-Tremblay

Tremblay, C., \& de Oliviera Jayme, B. (2015). Community knowledge cocreation through participatory video. Action Research. 13 ,3, 298-314. https:// doi.org/ 0.1177/1476750315572158

Ward, D. (2003). Where has real group work gone? Reasserting the fundamentals. In J. Lindsay, D. Turcotte, and E. Hopmeyer (eds). Crossing Boundaries and Developing Alliances Through Group Work. (pp. 53-58). New York: Haworth.

Wood, L. \& McAteer, M. (2017). Levelling the playing fields in PAR: The intricacies of power, privilege and participation in a university-communityschool partnership. Adult Education Quarterly. 67, 4, 251-165. https://doi-org. ezproxy.auckland.ac.nz/10.1177/0741713617706541

Wood, L., Greyling, L \& de Toit, P. (2015). Editorial: Descending from the ivory tower: The challenges and successes of community engagement for mutual learning. Educational Research for Social Change (ERSC). 4 ,1, 1-4. http://ersc. nmmu.ac.za/articles/ERSC_Vol_4_No_1_ALL_ARTICLES_April_2015_ as_on_20_April_2015B.pdf - page=5

Wright, R. (2017). To what extent are people with intellectual disabilities (ID) active partners in focus group research? A literature review. Groupwork. 26, 1, 59-75. https://doi.org/10.1921/gpwk.v26il.982 